Trauma Berufskrankh $2006 \cdot 8$ [Suppl 3]:

S283-S285

DOI 10.1007/s10039-006-1141-z

Online publiziert: 17. Mai 2006

(c) Springer Medizin Verlag 2006

\author{
R. Kübke \\ Berlin
}

\title{
Rolle des beratendes Arztes
}

\section{Aus Sicht eines nebenamtlich beratenden Arztes}

\begin{abstract}
Nicht immer ist man sich bei Übernahme eines Vortrags sofort bewusst, welche auch Zeit füllenden Dimensionen sich bei näherer Betrachtung des zugedachten Themas ergeben. So hatte ich meine Rolle als Beratungsarzt bisher nie ernsthaft durchdacht, sondern immer nur nach bestem Wissen und Gewissen auszufüllen gesucht. Und nun sollte ich diese Rolle gegenüber hauptamtlichen Kollegen abgrenzen:

- Worauf wollen die Kongressveranstalter hinaus?

- Was interessiert die Zuhörer an dieser Pro-und-Kontra-Thematik?
\end{abstract}

Letztendlich musste ich mir meiner Rolle als niedergelassener D-Arzt und nebenamtlicher Beratungsarzt neu bewusst werden.

\section{Tätigkeitsprofil}

Der Beratungsarzt dient als Schnittstelle und Übersetzer medizinischer Tatbestände zwischen nichtärztlichen Mitarbeitern der gesetzlichen Unfallversicherung, den klinisch ambulant tätigen $\mathrm{H}$ - und DÄrzten, den stationär tätigen Durchgangsärzten sowie allen am bg-lichen Heilverfahren beteiligten Leistungserbringern und natürlich auch den Versicherten.

\section{Voraussetzungen für die Arbeit als nebenamtlicher Beratungsarzt}

Grundvoraussetzung ist, dass es sich um einen erfahrenen Durchgangsarzt mit langjähriger klinischer und praktischer Tätigkeit handelt. Wesentlicher weiterer Punkt ist, dass es bei Betreibung einer Gemeinschaftspraxis gewährleistet ist, dass einer der beiden Partner Beratungen persönlich wahrnehmen kann, insbesondere wenn er von der beratenden Berufsgenossenschaft gebeten wird, vor Ort in der Bezirksverwaltung tätig zu sein. Gleiche Vertretungsvoraussetzungen gelten auch für die klinisch tätigen Kollegen. Darüber hinaus ist es unabdingbar, regelmäßige Fortbildungsveranstaltungen zu besuchen und auch im kollegialen Umfeld sowohl in die Kliniken als auch zu den niedergelassenen Kollegen gute Kontakte zu haben.

Es gibt keinen rechtlichen Anspruch auf eine Tätigkeit als Beratungsarzt, sondern man wird von der Berufsgenossenschaft bzw. vom Landesverband gebeten bzw. gefragt.

\section{Rolle des Beratungsarztes}

\section{Aus Sicht der Verwaltung}

1. Der Beratungsarzt muss sich mit dem beratenden Sachbearbeiter bei der Steuerung und der Kontrolle des Heilverfahrens auseinandersetzen.

2. Er muss Gutachten überprüfen.

3. Er wird zur Rechnungsüberprüfung herangezogen und bei der Organisation der spezifischen Nachbehandlung gefragt.

4. Sein Wissen ist bei der Beratung zur Planung der Wiedereingliederung erwünscht.
Wesentlicher Teil der Tätigkeit sind die persönlichen Gespräche mit den Sachbearbeitern an der Akte, ergänzt um Fortbildungsveranstaltungen, Weiterbildungen und Schulung der Mitarbeiter.

\section{Beispielfälle}

Als Beispiel für die Notwendigkeit der Überprüfungen von Gutachten seien exemplarisch folgende Fälle vorgestellt:

Fall 1. In dem Gutachten zu diesem Fall (• Abb. 1) wurde nach einer vorderen Kreuzbandplastik behauptet, dass die Transplantatpositionierung regelrecht sei, der Patient zwar über ein Instabilitätsge-

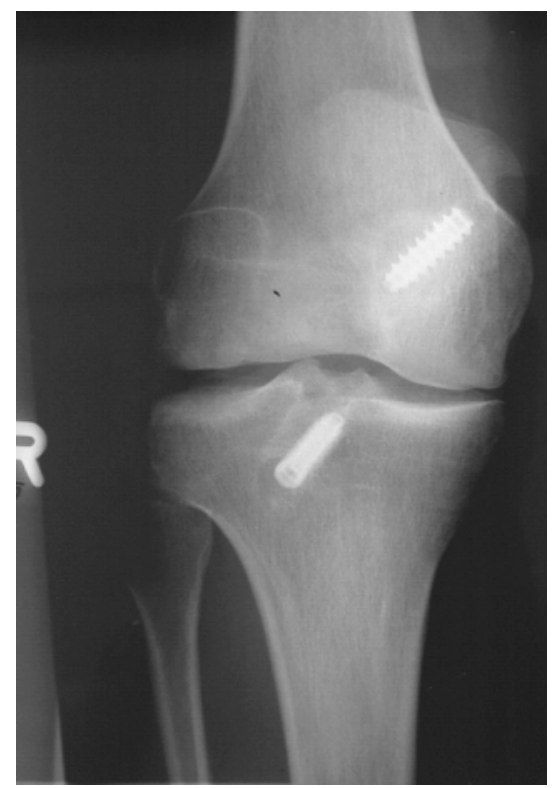

Abb. $1 \Delta$ Fehlpositioniertes vorderes Kreuzbandtransplantat 


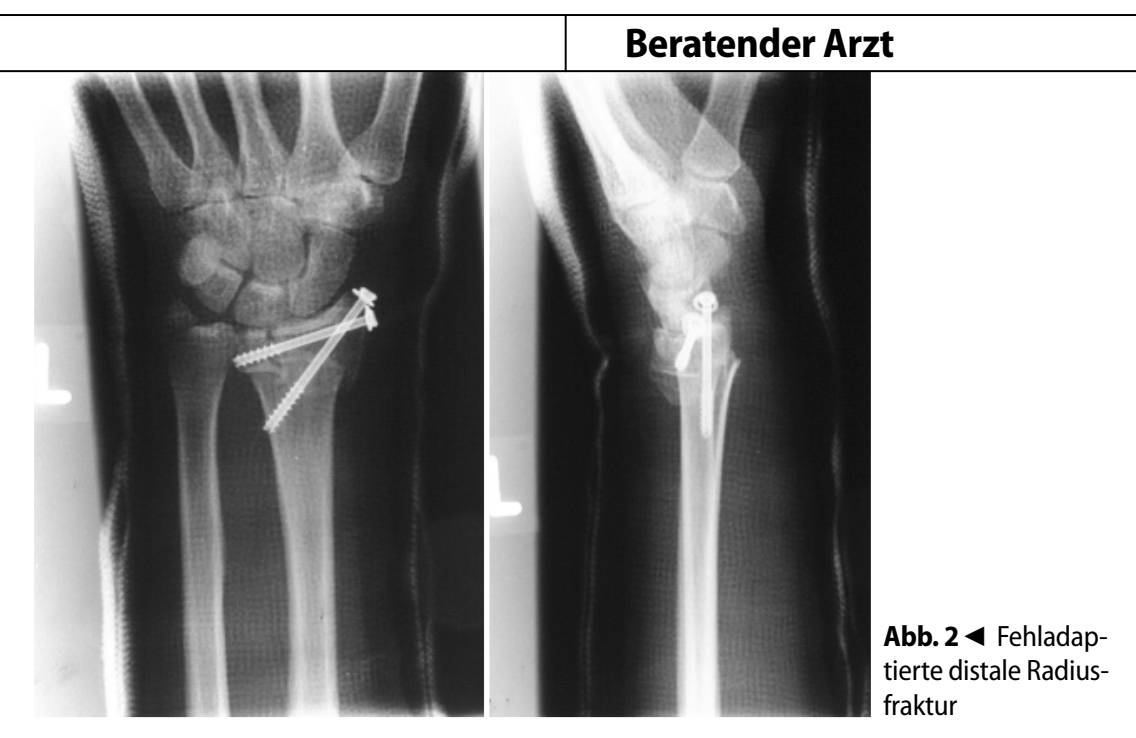

fühl klage, aber eigentlich ansonsten keine Auffälligkeiten bestünden ...

Zur Vergegenwärtigung: Das Röntgenbild zeigt eine komplette Fehlpositionierung des proximalen Blocks, der wie bei einer hinteren Kreuzbandplastik medial positioniert ist. Auch die distale Verankerung tibial ist völlig fehlpositioniert. Erst durch Intervention konnte dem Verletzten, der schon an seinem eigenen Krankheitswert gezweifelt hatte, dessen anhaltende Klagen sowie die Behandlungsbedürftigkeit aber medizinisch berechtigt waren, mit einer erneuten Operation und Nachuntersuchung geholfen werden. Dieses Beispiel zeigt, wie wichtig der erfahrene ärztliche 2. Blick ist.

Dass die Verwaltung in diesem Fall an Regress dachte, ist verständlich, aber es handelte sich eindeutig um ein haftungsrechtliches, kein beratungsärztliches Problem.

Fall 2. In Abb. $\mathbf{2}$ ist eine vollkommen fehladaptierte distale Radiusfraktur dargestellt, die durch persönliche Intervention des Beratungsarztes mit Übernahme der Heilverfahrenssteuerung letztendlich durch eine Reoperation zu einem befriedigenden Ergebnis geführt werden konnte. In solchen Fällen muss der Beratungsarzt unverzüglich mit den behandelnden Ärzten sprechen und ggf. die sofortige Zuweisung in ein Zentrum sicherstellen.

\section{Für den Versicherten}

Hier ist vordringlich die zeitgerechte Klärung, ob ein versicherter Unfall vorliegt, zu nennen (s. Fall 4). Zudem besteht die

\section{Beispielfälle}

Fall 3. Eine Patientin ging mit ihrem Kleinkind im Kinderwagen morgens einkaufen und wurde von einem psychisch schwer gestörten Täter angegriffen. Die Mutter beugte sich, um ihr Kind zu schützen, über den Kinderwagen und wurde mit insgesamt 34 Messerstichen in den Rücken von dem Täter verletzt. Nur durch zeitgerechte und glückliche Versorgung in einem nahe gelegenen Traumazentrum konnte die Patientin gerettet werden. In einem hervorragenden Zusammenhangsgutachten wurde eine Minderung der Erwerbsfähigkeit für die Patientin dauerhaft bewertet. Im Urteil aber wurde die persönliche Situation des Täters über 6 Seiten geschildert, die des Opfers über eine 1/2 Seite abgehandelt - das Kind wurde überhaupt nicht erwähnt.

Diese Hilfeleistung der Mutter ist bg-lich versichert!

Fall 4. Ein Arzt trank im Labor des Forschungshauses aus seiner eigenen Seltersflasche und erlitt lebensgefährliche Vergiftungen.

Hier war die Verwaltung nicht nur zu beraten, sondern auch der beratende Hinweis auf Einschaltung der Staatsanwaltschaft war wichtig.

\section{Aus Sicht der Kollegen}

Sie erwarten vom Beratungsarzt eine objektive Prüfung bei jeglichen Heilverfahrenskonflikten und eine kollegiale Rücksprache bei Unklarheiten im Gutachten sowie eine kollegiale Klärung bei unverstandener Therapie. Dies soll dazu führen, insbesondere auch bei der medizinisch relevanten Rechnungsüberprüfung deren Notwendigkeit und Wirtschaftlichkeit nicht als Schikane, sondern als objektive Prüfung verstehen zu helfen.

Da z. T., wie beim Autor, 2 Berufsgenossen beraten werden, deren Profile sehr unterschiedlich sind, muss auch beim Beratungsarzt eine große Flexibilität vorausgesetzt werden, damit seine Beratung von den Geschäftsführern geschätzt und akzeptiert wird. Im Fall des Autors fallen bei der einen Berufsgenossenschaft pro Jahr fast 100.000 Unfälle an, bei der anderen 
des Teilgewerbes 25.000, die jedoch ebenfalls eine hohe Kompetenz verlangen.

Darüber hinaus gibt es einige Vorurteile gegenüber Beratungsärzten, nach denen sie nie zur Verfügung stehen, wenn man sie braucht. Dieser Vorwurf ist als historisch zu betrachten, da in der modernen Kommunikationswelt jederzeit Kontakt aufgenommen werden kann.

Auch wird häufig vermutet, dass durch die enge Nähe des Beratungsarztes dieser nur im Sinne der von ihm beratenen Berufsgenossenschaft entscheidet. Gerade hier zeigt sich die besondere Rolle des nebenamtlich tätigen Beratungsarztes: Er ist wirtschaftlich durch seine freiberufliche, selbstständige Tätigkeit in der Praxis nicht so abhängig wie ein angestellter Arzt und kann entsprechend auch unbelastet Kontakte $\mathrm{zu}$ anderen Kollegen und Kliniken pflegen.

Abschließend möchte ich noch auf eine überaus interessante Internetseite verweisen: http://www.unfallopfer.de - hier haben Versicherte Meinungen zu behandelnden und begutachtenden Ärzten hinterlegt.

\section{Fazit}

Der nebenberuflich tätige Beratungsarzt wird täglich von Patienten und Kollegen in seiner praktischen klinischen Arbeit evaluiert. Auch die Verwaltungen beobachten sowohl seine ärztlichen als auch seine bg-lichen Fähigkeiten. Er ist ökonomisch vorrangig von ärztlicher Arbeit abhängig, und er kann aus täglichem Erleben die Brücke zwischen Theorie und Praxis realitätsnah schlagen. Aus der Sicht des Autors ist die Beteiligung nebenamtlicher Beratungsärzte auch zukünftig für ein erfolgreichen bg-liches Heilverfahren unverzichtbar.

\section{Korrespondierender Autor}

\section{R. Kübke}

Alt Tempelhof 43, 12103 Berlin

rkuebke@t-online.de

Interessenkonflikt. Keine Angaben

Trauma Berufskrankh 2006 · 8[Suppl 3]:S283-S285 DOI 10.1007/s10039-006-1141-z

(c) Springer Medizin Verlag 2006

R. Kübke

\section{Rolle des beratendes Arztes. Aus Sicht eines nebenamtlich beratenden Arztes}

\section{Zusammenfassung}

Der Beratungsarzt ist ein wichtiger Faktor in der Kommunikation zwischen gesetzlicher Unfallversicherung, Leistungserbringern und den Versicherten. Nebenamtlicher Beratungsarzt kann ein Durchgangsarzt mit langjähriger klinischer und praktischer Tätigkeit werden, der Partner einer Gemeinschaftspraxis oder Angestellter einer Klinik mit entsprechenden Vertretungsvoraussetzungen ist. Dies beinhaltet zum einen seine weitgehende ökonomische Unabhängigkeit, andererseits ist er auch zu einer realitätsnahen Beurteilung der einzelnen Fälle in der Lage. Wesentlicher Teil der Tätigkeit sind Gespräche mit den Sachbearbeitern an der Akte, Überprüfung von Gutachten, aber auch Rechnungen sowie die Hilfe bei der Organisation der spezifischen Nachbehandlung und der Planung

\section{The role of doctors retained by employers' liability insurance companies. The view of such a doctor also working in another medical position}

\section{Abstract}

The doctors retained by the employers' liability insurance companies are an important factor in communication between legally required accident insurance companies, service providers and insured persons. A doctor retained to do this work on a part-time basis as a second occupation can be a specialist in emergency medicine with many years of experience in hospital and private practice, a partner in a joint practice or a doctor employed in a hospital with appropriate arrangements for cover. On the one hand, this guarantees the doctor's financial independence, while in addition s/he is also in a position to assess individual cases by drawing on hands-on experience. Discussions with those responsible for working on the files, checks on the expert reports and also the invoices and help in the provision of specific aftertreatment and planning of the patients' re- der Wiedereingliederung. Zunehmend häufiger ist der Beratungsarzt als Mediator gefragt, um eine Eskalation von Konflikten vermeiden zu helfen. Manchmal ist auch sein besonderer Einsatz für die Rechte des Patienten gefragt! Der nebenberuflich tätige Beratungsarzt wird täglich von Patienten, Kollegen und der Verwaltung evaluiert. Wie aus vorliegendem Beitrag ersichtlich, ist er auch zukünftig für ein erfolgreichen bg-liches Heilverfahren unverzichtbar.

\section{Schlüsselwörter}

Nebenamtlicher Beratungsarzt · bg-liches Heilverfahren · Ökonomische Unabhängigkeit · Realitätsnahe Beurteilung · Konfliktdeeskalation

\begin{abstract}
integration make up an essential part of the work. Increasingly, a doctor retained by the employers' liability insurance companies is also required to act as a mediator to help avoid the escalation of any conflicts. Sometimes s/ he is also required to stand up specifically for patients' rights! Doctors working in this way are assessed daily by patients, colleagues and administrators. As seen from this paper, their work will remain essential in the future for successful treatment approved by employers' liability insurance companies.
\end{abstract}

\section{Keywords}

Doctors also retained for part-time consultations in insurance matters - Treatment approved by employers' liability insurance companies - Financial independence - Assessment drawing on background of appropriate experience $\cdot$ De-escalation of conflict 